\title{
Feasibility of circulating tumor cells (CTCs) combined with medical imaging examinations to screen for cancer and improve diagnosis in non-metastatic invasive breast cancer patients: a cohort study
}

\section{Xuan Shao \\ Department of Breast surgery, Second Affiliated Hospital, Zhejiang University School of Medicine https://orcid.org/0000-0003-1077-8089}

xiao yan jin

second affiliated hospital of zhejiang university school of medicine

zhi gang chen

second affiliated hospital of zhejiang university school of medicine

zhi gang zhang

second affiliated hospital of zhejiang university school of medicine

ke wang

second affiliated hospital of zhejiang university school of medicine

xiu yan yu

second affiliated hospital of zhejiang university school of medicine

wu zhen chen

Zhejiang University School of Medicine Second Affiliated Hospital

jing xin jiang

Zhejiang University School of Medicine Second Affiliated Hospital

zhen wang

Zhejiang University School of Medicine Second Affiliated Hospital

ying cui

hangzhou watson biotech inc

wan hung fan

hangzhou watson biotech inc

jian huang ( $\nabla$ drhuangjian@zju.edu.cn )

Department of Breast surgery, Second Affiliated Hospital, Zhejiang University School of Medicine https://orcid.org/0000-0003-3340-5007

\section{Research article}

Keywords: Circulating tumor cells, breast cancer, ultrasonography, mammography, magnetic resonance imaging, breast-specific gamma imaging

Posted Date: October 30th, 2020 
DOI: https://doi.org/10.21203/rs.3.rs-31589/v2

License: (c) (i) This work is licensed under a Creative Commons Attribution 4.0 International License. Read Full License 


\section{Abstract}

Background: Previous study has reported that circulating tumor cells (CTCs) could be served as a diagnostic biomarker in breast cancer (BC) screening. However, the differential efficacy of routine examination including ultrasound (US), mammogram (MG), magnetic resonance imaging (MR), and breast-specific gamma imaging (BSGI) and CTCs is unknown. This study aimed to compare CTCs with common used BC screening imaging modalities and to evaluate whether their combination would enhance the diagnostic potency in non-metastatic BC patients.

Methods: 102 treatment-naive non-metastatic BC patients, 177 patients with breast benign diseases (BBD) and 64 healthy females, who had CTC detection and at least one of the following medical imaging examinations, US, MG or MR between December 2017 and November 2018, were enrolled in this study.Correlations of CTC enumeration with patients' clinicopathological characteristics and medical imaging examinations were evaluated.

Results: CTC detection rates (average CTC counts) in stage I-III BC patients were 92.9\% (2.1), 87.2\% (2.4) and 100\% (4.2), respectively. CTCs counts were positively associated with cancer stage $(p=0.0084)$ and tumor size $(p=$ 0.0301). CTC counts were more correlated with US than MR or MG. CTC counts were not associated with molecular subtypes of BC nor breast-specific gamma imaging (BSGI) results, indicating that CTC enumeration cannot be used to predict molecular signatures of BC. CTCs and medical imaging examinations would have the best diagnostic performance for BC when CTC cut-off was set to 2 and imaging Breast Imaging-Reporting and Data System (BIRADS) was set to 4b. Combination of CTC with US, MG or MR increased the sensitivity for BC diagnosis, especially for MG. Sensitivity of MG increased from 0.694 to 0.917 , even more than in conjugation with US (0.901).

Conclusion: CTCs counts can be used as a diagnostic aid in BC screening and early diagnosis. CTCs counts were more relevant to US than MR or MG. Conjugation of CTCs counts would improve the diagnostic potency of medical imaging examinations for diagnosing $B C$, especially for $M G$ in Chinese women.

\section{Background}

In China, there were 304,000 newly diagnosed cases of breast cancer (BC) and approximately 69,900 women died from BC in 2015 [1]. According to the World Health Organization, although the mortality rates of BC in developed countries are decreasing, incidence and mortality rates of BC in developing countries such as China and India are still increasing [2]. "Early detection, early treatment" means that patients would have better survival outcomes if the tumors were diagnosed earlier. So various methods have been proposed as BC early diagnosis and screening tools, including breast palpation, medical imaging methods and blood-based assay.

Diagnostic imaging modalities recommended by American Joint Committee on Cancer (AJCC) for BC screening include mammography (MG), ultrasound (US) and magnetic resonance imaging (MR). MG uses low dose X-rays to examine lesions in the breast, allowing to view small calcification points, tumor in situ, and the structure of the breast, which are signs of early cancer lesions. Studies showed that overall sensitivity of MG for cancer screening was $87 \%$ [3]. Breast US is a non-invasive examination that uses high-frequency acoustic reflection to reveal tissue inside the breast. Breast US is often used in conjunction with MG to increase the sensitivity of BC detection for women at average risk. US can help us identifying whether the lumps found in MG is solid or filled with fluid. Although US is more accurate than MG in differentiating breast masses or cysts, it can not detect the small calcification points and is less sensitive to detect tumors which is less than $5 \mathrm{~mm}$ with diameter or deep in the breast. MR is often used in conjunction with MG for high-risk women BC screening. In this article, MR refers to 
enhancing-MR. MR has no radiation exposure, and can provide excellent images with high contrast and resolution under appropriate conditions. MR is the most sensitive diagnostic tool for breast diseases, because it can be performed in any direction without the influence of tissue overlap or breast composition. MR is often used in the diagnosing and staging process rather than screening of BC. However, MR is more expensive than MG and US. Therefore, MG and US are the two most common used BC screening modalities in clinical practice in China. Breast specific gamma imaging (BSGI) is a physiologic and molecular approach to breast imaging using a high resolution, small-gamma camera and a tracer agent called 99mTc-Sestamibi (MIBI) which we have investigated in our previous study [4].

Circulating tumor cells (CTCs) are tumor cells which have detached from the primary tumor or metastatic sites into circulation. Studies have reported the prognostic value of CTC in BC [5-7]. Therefore, in the 8th edition of the AJCC cancer guidelines, it is written that CTC can be used as a prognostic biomarker in BC to predict patients' survival outcomes. Patients with metastatic breast cancer (MBC) usually have more CTC and BC patients with more CTC usually have shorter progression free survival and overall survival [6-7]. Moreover, CTC can reflect the tumor burden, and can be used as a monitoring biomarker to assess patients' response to treatment and tumor recurrences [8]. Previous study using CytoSorter ${ }^{\circledR}$ (Hangzhou Watson Biotech, Hangzhou, China) in BC has suggested that CTCs could be used as a diagnostic aid in early diagnosis and screening of BC [9].

Both CTCs and medical imaging examinations could be used as a screening method for BC. Therefore, the aims of this work were: (1) to correlate CTCs with medical imaging examinations, such as US, MG, MR or BSGI, and (2) to compare their diagnostic performances in $\mathrm{BC}$ diagnosis. This was a retrospective study. Non-metastatic BC patients, patients with benign breast diseases (BBDs) and healthy females, who have performed CTC detection and at least one of the following medical imaging examinations, US, MG or MR, BSGI, before treatment, were enrolled in this study. Correlations of CTCs with patients' clinicopathological characteristics and medical imaging examinations were evaluated to answer our questions.

\section{Methods}

\section{Patients and ethics}

This study followed the principles established in the Declaration of Helsinki and was approved by the ethics committee of Second Affiliated Hospital of Zhejiang University School of Medicine with a IRB number "(2018) lunshenshijidi (013) hao". Written consent for participation in this research and publication of their case details was obtained from each participant. In total, 102 non-metastatic BC patients and 241 controls (177 patients with BBDs and 64 healthy females), who had CTC detection and at least one of the following medical imaging examinations, US, MG or MR, BSGI, between December 2017 and November 2018 were included in this study. Inclusion criteria was as follows: (1) patients aged between 18 and 75 years old; (2) patients had CTC detection before treatment; (3) patients had no pathological proof of metastatic BC; (4) patients received at least once US, MG or MR, examination before treatment. Exclusion criteria was as follows: (1) patients had other malignant tumors or other malignant diseases within 5 years prior to enrollment; (2) patients had no medical imaging examination. Among the $102 \mathrm{BC}$ patients, 101 had US, 84 had MG, 91 had MR, while 42 had BSGI examinations. Among the 241 controls, 240 had US, 106 had MG, while 6 had MR examinations. Patients' clinicopathological characteristics, including age, histological type, grade, hormone receptors, human epidermal growth factor receptor 2 (HER2) and the cancer stage, and imaging examination results were collected. For the controls, only imaging BI-RADS scores were collected. 


\section{CTCs detection}

CTC detection was performed using CytoSorter ${ }^{\circledR}$ system with the epithelial cells detection kit. CTC detection procedure was described as in the previous study [9]. CTCs were identified as pan-cytokeratin-fluorescein isothiocyanate (PanCK-FITC) positive, cluster of differentiation 45-phycoerythrin (CD45-PE) negative, and DAPI (4',6-diamidino-2-phenylindole) positive cells.

\section{Medical imaging examinations and pathology}

US examination was performed with IU Elite ${ }^{\circledR}$ (Philips Healthcare, Best, Netherlands). MG examination was performed using Selenia ${ }^{\circledR}$ (Hologic, Santiago, USA). Breast MR examination was performed on a $1.5 \mathrm{~T}$ system with Aera ${ }^{\circledR}$ (Siemens, München, Germany). BSGI examination was performed with Dilon 6800 Gamma Camera (Dilon Technologies, Newport News, VA). All the different imaging data (US, MG, MR, BSGI) presented in this paper were independently reviewed by imaging specialists in their respective specialties (US, MG, MR, BSGI) and rated according to the latest BI-RADS version of their respective specialties except BSGI which is according to our previous study. BI-RADS is a risk assessment of likelihoods of BC, with a score ranging between 0 and 6 . A score of 0 indicates an incomplete test. Categories 1-6 represent negative, benign, probably benign, suspicious or indeterminate abnormality, highly suggestive of malignancy, and known cancer, respectively. Category 4 is subdivided into 3 sub-categories: 4A, 4B and 4C for a low, moderate and high suspicion of malignancy, respectively. Breast density was visually estimated according to BI-RADS classification and classified as follows [10]: $a=$ The breasts are almost entirely fatty. $b=$ There are scattered areas of fibroglandular density. $c=$ The breasts are heterogeneously dense, which may obscure small masses. $d=$ The breasts are extremely dense, which lowers the sensitivity of mammography. Detailed examination procedure for BSGI and the cut-off value was described as in the previous study [4]. Pathology was reviewed by pathologists independently and classified according to the 2012 edition of WHO classification of tumours of the breast and divided into three categories, namely micro-invasive breast cancer, invasive breast cancer and non-invasive breast cancer. The staging system for breast cancer is conducted according to AJCC/UICC edition 8th.

\section{Statistical analysis}

Statistical analyses were performed using Prism 6.0 (Graphpad, La Jolla, CA, USA). Student t test was used for continuous variables, as appropriate. The $x^{2}$ test and Fisher's exact test were used for the comparison of categorical parameters. One-way analysis of variance (ANOVA) was performed to calculate the differences among multiple groups. The cut-off values of CTC, US, MG and MR for BC diagnosis were determined by Youden index (sensitivity + specificity -1). CTC counts or imaging BI-RADS no less than cut-off value was defined as CTC or imaging positive. The ROC curves were plotted to evaluate the diagnostic potencies of CTC, US, MG and MR for diagnosing BC. A two-sided $p$ value less than 0.05 was considered statistically significant.

\section{Results}

\section{Comparison of CTCs with BC patients' clinicopathological features}

Studies have shown that CTCs could be used as a diagnostic aid to distinquish BC patients from the non-tumor people and reflect tumor burden [5, 8]. 102 BC patients, including 42 stage I, 47 Stage II and 13 stage III, along with 177 patients with BBD and 64 healthy females were enrolled in this study. CTCs showed a statistical significance among BC patients, patients with BBD and healthy control as shown in Table $1(p<0.0001)$.. The CTC detection 
rates (average CTC counts) for healthy volunteers, patients with BBD and BC patients were $17.2 \%(0.2), 40.7 \%(0.5)$ and $91.2 \%$ (2.5), respectively. To determine whether CTCs were associated with BC patients' clinicopathological characteristics, statistical analyses were performed among different groups of BC patients based on their age, AJCC stages, tumor size, lymph node metastasis, molecular subtypes and histology grades. CTC detection rates (average CTC counts) in stage I-III BC patients were 92.9\% (2.1), 87.2\% (2.4) and 100\% (4.2), respectively. CTCs were correlated with AJCC stage $(p=0.0084)$. Furthermore, CTCs were associated with tumor size as well $(p=0.0301)$, but not with age, lymph node metastasis, molecular subtype, or histology grade. If BC patients were grouped first by the molecular subtype, no correlation of CTCs with AJCC stage would be observed in each molecular subtype group (data not shown).

\section{Comparison of CTCS with US}

101 out of 102 BC patients and 240 out of 241 controls had US examination. Results of US were grouped according to the tumor size estimated by US or to BI-RADS as shown in Table 2. Based on the tumor size $T$, five categories were defined - 1a, 1b, 1c, 2 and 3, representing patients with $T \leq 0.5 \mathrm{~cm}, 0.5 \mathrm{~cm}<T \leq 1 \mathrm{~cm}, 1 \mathrm{~cm}<T \leq 2 \mathrm{~cm}, 2 \mathrm{~cm}$ $<T \leq 5 \mathrm{~cm}$, and $T>5 \mathrm{~cm}$, respectively. CTC enumerations were correlated with US when BC patients were grouped into 1,2 and 3 or into $1 \mathrm{a}+1 \mathrm{~b}, 1 \mathrm{c}, 2$ and $3(p=0.0322$ or 0.0309$)$. CTC enumerations were correlated with US as well when BC patients were grouped by BI-RADS $(p<0.0001)$. More CTCs would be detected in patients with bigger tumors or higher BI-RADS scores. Taken together, CTCs were positively associated with US examination.

\section{Comparison of CTCs with MG, MR and BSGI in BC}

84 and 91 out of 102 BC patients, and 106 and 6 out of 241 controls, had MG and MR examinations, respectively. Breast tissue composition category was used to measure the density of breast tissue. Comparisons of CTCs with MG, and MR were shown in Table 3 and 4, respectively. CTCs were not correlated with tumor size nor breast tissue composition category evaluated by MG or MR (all $p>0.05$ ). However, CTCs showed statistically significant correlations with MG BI-RADS $(p<0.0001)$ and MR BI-RADS $(p<0.05)$. No correlation was found between CTCs and MR time-signal intensity curves (TIC) $(p>0.05)$. 42 BC patients had BSGI examination and no ststistically significant correlation between CTCs and BSGI was found as shown in Table 5.

\section{Comparison of CTCs with medical imaging examinations with cut-off settings}

Youden index was used to dtermine the cut-off value of CTCs and cut-off scores of BI-RADS at which it would have the best performance for diagnosing BC. When CTC cut-off value and US, MG and MR BI-RADS scores were set to 2 and $4 \mathrm{~b}$, it would generate the highest Youden index of $0.71,0.69,0.6$ and 0.71 , respectively. Therefore, next we would like to know whether CTCs would be more relevant to medical imaging examinations when the cut-off was used. CTC or BI-RADS above cut-off values was defined positive. As shown in Table 6, the positive rates of CTCs, US, MG and MR could all differentiate BC patients from the control (all $p<0.05$ ). CTC positive rate was only correlated with tumor histological grade $(p=0.029)$, while US positive rates were correlated with AJCC stage and lymph node metastasis (both $p<0.05)$. MR positive rate was only correlated with AJCC stage $(p=0.0422)$. CTC and MG positive rates were not associated with BC patients' clinicopathological features. Correlation of CTC positive rates with US, MG and MR are shown in Table 2-4, respectively. CTC positive rate showed significant correlations with US, MG and MR BI-RADS (all $p<0.05$ ). When both CTC and medical imaging examination cut-off values were used, CTC was correlated with US, MR and MG as well (all $p<0.05$ ). Due to the small number of control who had MR examination, MR has a higher $P$ value compared to US and MG. 
Diagnostic performances of CTCs, US, MG and MR for BC were listed in Table 7. Among the 4 methods, MR has the highest sensitivity of 0.872 , while CTC has the highest specificity of 0.938 . CTC has the highest accuracy of 0.889 . followed by MR of 0.87 , US of 0.848 and MG of 0.82 . Based on the AUC, US has the best diagnostic performance, followed by CTC, MG and MR. All AUC are greater than 0.8, indicating that CTC, US, MG and MR all have excellent discriminatory abilities for BC diagnosis. Conjugation of CTCs with medical imaging examinations increased the sensitivity of US, MG and MR to $0.921,0.917$ and 0.942 , respectively. The dianostic performance for BC of combing CTCs with medical imaging examination was better than the combination of any two of the medical imaging examinations.

\section{Discussions}

Previous study with CytoSorter ${ }^{\circledR}$ in BC showed that CTC could reflect tumor burden and could be used as a diagnostic aid in BC screening and early diagnosis [9]. First we compared CTCs with enrolled subjects' clinicopathological features and found that CTCs could distinguish BC patients from the control and were associated with cancer stage and tumor size, but not with lymph node metastasis, patients' age and tumor molecular subtype and histology grade. A recent study shows that mesenchymal type of CTCs in BC is more invasive and more related with lymph node and distant metastasis [11], thus more mesenchymal CTCs would be found in late cancer patients. There are many methods to detect CTCs in practice, and for the methods based on biological properties of CTCs, epithelial cell adhesion molecule (EpCAM) antibody is the most common used antibody to capture CTCs. It is reported that CTCs can reflect tumor burden and is positively associated with cancer stage and tumor size. We used (EpCAM) antibody to capture CTCs, as well in the study, and results showed CTCs were correlated with tumor size and cancer stage, but not with lymph node involvement. We figure that epithelial type of CTCs captured by EpCAM antibody are more associated with tumor size (T satge), while mesenchymal CTCs should be more associated with invasiveness. Youden index analysis showed that CTCs would have the best diagnostic potency for BC when CTC cut-off value was set to 2. If CTC positive was defined as any patient with CTC $\geqq 2$, CTC positive rates could still differentiate BC patients from the control, but were not correlated with tumor stage, tumor size, lymph node involvement, and tumor molecular subtypes as shown in Table 6. CTC positive rates were $71.4 \%, 73.4 \%$ and $74.2 \%$ in patients at I, T1 and N0 stage, respectively. High CTC positive rates already in early stage BC can explain why there was no statistical significance to be found among different TNM groups of BC patients concerning CTC positive rates.A trend of more CTCs in patients at advanced stage of disease was still found. CTC detection rates (positive rates) in stage I and II BC were 92.9\% (71.4\%) and 87.2\% (76.6\%), respectively, which is in line with the previous finding that CTC can be used as an screening aid for early stage BC [9].

Next, we tried to find correlation between CTCs with imaging methods and compared their diagnostic performance for BC. Our analysis showed CTCs were more associated with US and US performed best for diagnosing BC. Statistics results showed only CTCs and US were correlated with BC patients' clinicopathological features, which might explain why CTCs are more associated with US. Although MR is considered the most sensitive method for detection of $\mathrm{BC}, \mathrm{MR}$ is not used as the first line of imaging modality for $\mathrm{BC}$ screening in practice. Breast MR is more used in pretherapeutic BC staging, monitoring of primary systemic therapies and solving problematic diagnostic situations where direct biopsy is not possible. MR is a costly examination and usually not covered by the health insurrance. Therefore, most patients with BBD or healthy people would not perform breast MR in practice. Only 6 people from the control group had MR examination. The MR control group was too small and that could be the 
reason why MR had the lowest specificty of 0.833 . Thus, MR is removed from the discussion concerning diagnostic performance.

Comparing the diagnostic performances of CTCs and medical imaging examinations for BC, we found that MR had the highest sensitivity of 0.872 , while CTCs had the highest specificity of 0.938 . Although MR had the highest sensitivity, it also had the lowest specificity of 0.833 , which might be due to the low number of MR control group. Although MG is currently the only BC screening method shown to reduce mortality and the most efficient screening method recommended by American Cancer Society (ACS) and AJCC to detect early BC, it has the lowest sensitivity of 0.694 in our study. The sensitivity of MG depends on the patient's age and breast composition. Usually, sensitivity of MG should be higher in women over 50 and with fatty breasts [12]. The median age of enrolled BC patients is 53.4 years. Still MG showed the lowest sensitivity in this study. It could be due to that oriental women usually have dense breasts [13], therefore, MG was less sensitive in our study.

MG was not associated with any of BC patients' clinicopathological features, which may explain why CTCs were not correlated with the MG. We think the reason that MG was not associated with any clinicopathological features of BC in this study is due to the dense breast of Asian women [14]. MG usually has a sensitivity higher than 0.85 for breast cancer screening, but the sensitivity of MG in this study is only 0.694 . Studies have suggested that the dense breast causes the low sensitivity of MG in Asian women. Usually aged women have more fat breast. Liu et al has shown that MG has a higher sensitivity in aged women and for Chinese women younger than 55 years old, the diagnostic efficiency of breast cancer screening imaging examinations may be potentially improved by combining screening MG with US [15]. The average (median) age of enrolled breast cancer patients is 53.4 (55) years old. If only patients older than 55 years old were included for analysis, we found that MG was correlated with lymph node metastasis ( $p$ $=0.0168$ ). However, we could not analyze the correlation of MG with other breast cancer patients'clinicopathological features due to the low number of samples in each group. But if the analysis was performed based on the proportion, we found that MG was correlated with cancer stage as well with a p value smaller than 0.0001. Taken together, the dense breast should be the reason that MG was not associated with any clinicopathological features of $\mathrm{BC}$ in this study.

US can differentiate fluid-filled cysts from solid masses, therefore, it is most useful for looking at some breast changes, such as lumps. Studies show that BC detection rate with US is comparable with MG, with a greater proportion of invasive and node-negative cancers among US detections [16,17]. Adjunctive US to MG can increase the sensitivity and detection rate of early BC in younger women with dense breast. Since Chinese women usually have dense breast and only non-metastasis BC patients were enrolled in this study, US should be more sensitive than MG for BC detection. Our rsults show that US has a higher sensitivity of 0.842 than CTC (0.775) and MG (0.694). As shown in Table 6, US was correlated with cancer stage and lymph node metastasis, which might explain why CTCs were more associated with US since CTCs were associated with cancer stage as well. US usually generates more false-positive results [18], which can be confirmed in our study by the lower specificity of US (0.85) compared to those of CTCs (0.938) and MG (0.906) as shown in Table 7.

BSGI is a molecular breast imaging procedure that shows the metabolic activity of breast lesions [19]. MR-TIC can reflects the hemodynamic features of a specific lesion, especially about the vascular structure around tumor. CTCs were not associated with BSGI nor MR-TIC. Bansal et al used flow cytometry to detect CTCs in 114 BC patients and found that CTC positivity did not show any correlation with the tumor immunohistochemical profile [20]. Taken together, CTC enumeration cannot be used as a biomarker to reflect the molecular signature of BC. CTCs are heterogeneous. Some are epithelial type, while some are mesenchymal type. Some may express stem-cell markers, 
such as CD44, or other proteins, such as PD-L1 or HER2. Therefore, profiling of CTCs is considered as a promising tool to reveal the molecular signature of $\mathrm{BC}$, allowing for a better personal treatment rearrangement.

Our results show CTCs are more associated with US than MG or MR. Comparing the consistence between CTCs and medical imaging examinations in BC detection, CTCs show the highest consistence of $70.3 \%$ with US, followed by $68.2 \%$ with MR and $58.3 \%$ with MG, which may explain why CTCs are more correlated with US than MR, or MG. Interpretation of medical imaging examinations require a radiologist's expertise. An inexperienced expert might misinterpret the imagings to generate false results. Compared to CTCs, medical imaging examinations might be considered as a diagnostic tool with more human error bias.

Lastly, we liked to know whether conjugation of CTC would improve the sensitivity of imaging examinations for BC. Table 7 showed that combination of CTC with any of the medical imaging examinations increased the sensitivity for BC diagnosis, especially for MG. Sensitivity of MG increased from 0.694 to 0.917 , even more than in conjugation with US (0.901). Medical imaging examinations combine with CTCs would improve their diagnostic potency for BC

\section{Conclusions}

Results of this work showed that CTCs detected by CytoSorter ${ }^{\circledR}$ system could be used as a diagnostic aid in assistance of early diagnosis and screening of BC. CTCs were more relevant to US than MG or MR. Conjugation of CTCs would improve the diagnostic potency of medical imaging modalities for BC diagnosis, especially for MG. Still more studies on larger patient population should be conducted to confirm our findings and to elucidate the clinical value of utilizing CTCs as a diagnostic, therapeutic and prognostic indicator in BC.

\section{Abbreviations}

American Cancer Society (ACS)

American College of Radiology (ACR)

American Joint Committee on Cancer (AJCC)

breast cancer $(\mathrm{BC})$

Breast Imaging-Reporting and Data System (BI-RADS)

breast-specific gamma imaging (BSGI)

circulating tumor cells (CTCs)

epithelial cell adhesion molecule (EpCAM)

false negative rates (FNR)

false-positive results (FPR)

human epidermal growth factor receptor 2 (HER2)

mammogram (MG) 
magnetic resonance imaging (MR)

99mTc-Sestamibi (MIBI)

metastatic breast cancer (MBC)

One-way analysis of variance (ANOVA)

time-signal intensity curves (TIC)

ultrasound (US)

\section{Declarations}

\section{Ethics approval and consent to participate}

This study followed the principles established in the Declaration of Helsinki and was approved by the ethics committee of Second Affiliated Hospital of Zhejiang University School of Medicine which had obtain JCl certification. Written informed consent was obtained from individual or guardian participants.

\section{Consent for publication}

Not applicable.

\section{Availability of data and material}

All data analysed during this study are included in this published article.

\section{Competing interests}

The authors declared that they had no competing interests.

\section{Funding}

This study was supported in part by grants from Research and Application of 3D Printing Integrated Manufacturing Technology of Personalized Medical AIDS, Zhejiang Science and Technology Department (grant number 2017C01020). Jian Huang is the main principal of the funder and the others are attending the project except $Y \mathrm{C}$ and WHF.

\section{Authors' contributions}

Conception and design: $X S$ and $\mathrm{J} \mathrm{H}$. Acquisition of data: $X Y$ J, ZG C, K W, XY Y, WZ C, JX J, Z W. Analysis and interpretation of data: X S , ZG Z, Y C, WH F and J H. Writing of the manuscript: X S, WH F , ZG Z. Review of the manuscript: All authors have read and approved the manuscript.

\section{Acknowledgements}

We would like to thank the CTC Laboratory at Hangzhou Watson Biotech, Inc. (Hangzhou, China) for their technical help in CTC detection, and the Department of Radiology in our hospital for their help in medical imaging examinations. 


\section{References}

[1] Zheng R, Sun K, Zhang S, Zeng H, Zou X, Chen R, et al. Report of cancer epidemiology in China, 2015. Chin J Oncol 2019;41(1):19-28

[2] Siegel RL, Miller KD, Jemal A. Cancer statistics, 2019. CA Cancer J Clin 2019;69(1):7-34

[3] Breast Cancer Surveillance Consortium (BCSC). Performance measures for 1,838,372 screening mammography examinations from 2004 to 2008 by age - based on BCSC data through 2009. National Cancer Institute. 2016; https://www.bcsc-research.org/statistics/mammography_data

[4] Yu X, Hu G, Zhang Z, Qiu F, Shao X, Wang X, et al. Retrospective and comparative analysis of (99m)Tc-Sestamibi breast specific gamma imaging versus mammography, ultrasound, and magnetic resonance imaging for the detection of breast cancer in Chinese women. BMC Cancer. 2016 Jul 11;16:450.

[5] Harigopal M, Kowalski D, Vosoughi A. Enumeration and molecular characterization of circulating tumor cells as an innovative tool for companion diagnostics in breast cancer [published online ahead of print, 2020 Jun 30]. Expert Rev Mol Diagn. 2020;10.1080/14737159.2020.1784009.

[6] Bidard FC, Proudhon C, Pierga JY. Circulating tumor cells in breast cancer. Mol Oncol. 2016;10(3):418-30

[7] Hayes DF, Cristofanilli M, Budd GT, Ellis MJ, Stopeck A, Miller MC, et al. Circulating tumor cells at each follow-up time point during therapy of metastatic breast cancer patients predict progression-free and overall survival. Clin Cancer Res. 2006;12(14 Pt 1):4218-24

[8] Yan WT, Cui X, Chen Q, Li YF, Cui YH, Wang Y, et al. Circulating tumor cell status monitors the treatment responses in breast cancer patients: a meta-analysis. Sci Rep. 2017;7:43464

[9] Jin L, Zhao W, Zhang J, Chen W, Xie T, Wang L, et al. Evaluation of the diagnostic value of circulating tumor cells with CytoSorter ${ }^{\circledR}$ CTC capture system in patients with breast cancer. Cancer Med. 2020 Mar;9(5):1638-1647.

[10] D.A. Spak, J.S. Plaxco, L. Santiago, M.J. Dryden, B.E. Dogan.BI-RADS® fifth edition: A summary of changes. Diagnostic and Interventional Imaging (2017) 98, 179-190.

[11] Zhou J, Zhu X, Wu S, Guo J, Zhang K, Xu C, et al. Epithelial-mesenchymal transition status of circulating tumor cells in breast cancer and its clinical relevance. Cancer Biol Med. 2020 Feb 15;17(1):169-180.

[12] Nazari SS, Mukherjee P. An overview of mammographic density and its association with breast cancer. Breast Cancer. 2018 May;25(3):259-267.

[13] Buist DS, Porter PL, Lehman C, Taplin SH, White E. Factors contributing to mammography failure in women aged 40-49 years. J Natl Cancer Inst. 2004 Oct 6;96(19):1432-40.

[14] Ohuchi N, Suzuki A, Sobue T, Kawai M, et al. J-START investigator groups. Sensitivity and specificity of mammography and adjunctive ultrasonography to screen for breast cancer in the Japan Strategic Anti-cancer Randomized Trial (J-START): a randomised controlled trial. Lancet. 2016 Jan 23;387(10016):341-348.

[15] Liu J, Liu PF, Li JN, Qing C, Ji Y, Hao XS, Zhang XN. Analysis of mammographic breast density in a group of screening chinese women and breast cancer patients. Asian Pac J Cancer Prev. 2014;15(15):6411-4. 
[16] Shen S, Zhou Y, Xu Y, et al. A multi-centre randomised trial comparing ultrasound vs mammography for screening breast cancer in high-risk Chinese women. Br J Cancer. 2015;112(6):998-1004.

[17] Badu-Peprah A, Adu-Sarkodie Y. Accuracy of clinical diagnosis, mammography and ultrasonography in preoperative assessment of breast cancer. Ghana Med J. 2018 Sep;52(3):133-139.

[18] Berg WA, Bandos Al, Mendelson EB, Lehrer D, Jong RA, Pisano ED. Ultrasound as the Primary Screening Test for Breast Cancer: Analysis From ACRIN 6666. J Natl Cancer Inst. 2015 Dec 28;108(4):djv367.

[19] Huppe Al, Mehta AK, Brem RF. Molecular Breast Imaging: A Comprehensive Review. Semin Ultrasound CT MR. 2018 Feb;39(1):60-69.

[20] Bansal C, Pujani M, Misra S, Srivastava AN, Singh US. Circulating Tumor Cells in Breast Cancer: Correlation with Clinicopathological Parameters, Hormone Profile and MicroRNA Polymorphisms. Turk Patoloji Derg.

2016;32(3):148-57

\section{Tables}

Table 1. Correlation of CTCs with BC patients' clinicopathological features 


\begin{tabular}{|c|c|c|c|c|c|c|c|c|c|}
\hline Group & $\mathrm{n}$ & $\begin{array}{l}\text { Average Age } \\
\text { ((Median, } \\
\text { years) }\end{array}$ & $\begin{array}{l}\text { CTC } \\
\text { Detected }\end{array}$ & $\begin{array}{l}\text { CTC } \\
\text { Detection } \\
\text { Rate (\%) }\end{array}$ & $\begin{array}{l}\text { Average } \\
\text { CTC Count } \\
\text { (Range) }\end{array}$ & $P$ & $\begin{array}{l}\text { CTCs } \\
\geq 2\end{array}$ & $\begin{array}{l}\text { CTCs } \\
<2\end{array}$ & $P$ \\
\hline \multicolumn{10}{|c|}{$(n=102+241)$} \\
\hline BC & 102 & $53.4(55)$ & 93 & 91.2 & $2.5(0-15)$ & \multirow{3}{*}{$<.0001$} & 79 & 23 & \multirow{3}{*}{$\dot{0} 0001$} \\
\hline BBD & 177 & $43.5(44)$ & 72 & 40.7 & $0.5(0-4)$ & & 15 & 162 & \\
\hline Healthy & 64 & $40.6(36.5)$ & 11 & 17.2 & $0.2(0-1)$ & & 64 & 0 & \\
\hline \multicolumn{10}{|c|}{ (BC Patients $\mathrm{n}=102$ ) } \\
\hline \multicolumn{10}{|c|}{ AJCC Stage } \\
\hline । & 42 & $56(57)$ & 39 & 92.9 & $2.1(0-7)$ & \multirow[t]{3}{*}{0.0084} & 30 & 12 & \multirow[t]{3}{*}{0.0964} \\
\hline ॥ & 47 & $52.1(52)$ & 41 & 87.2 & $2.4(0-13)$ & & 36 & 11 & \\
\hline III & 13 & $53.1(55)$ & 13 & 100 & $4.2(2-15)$ & & 13 & 0 & \\
\hline \multicolumn{10}{|c|}{ TNM T Stage } \\
\hline T1 & 64 & $54.2(55.5)$ & 58 & 90.6 & $2.2(0-13)$ & \multirow[t]{3}{*}{0.0301} & 47 & 17 & \multirow[t]{3}{*}{0.2417} \\
\hline T2 & 32 & $52.1(50)$ & 30 & 93.8 & $3.1(0-15)$ & & 28 & 4 & \\
\hline T3 & 6 & $59(57.5)$ & 5 & 83.3 & $2.3(0-5)$ & & 4 & 2 & \\
\hline \multicolumn{10}{|c|}{ TNM N Stage } \\
\hline No & 62 & $54.9(56)$ & 56 & 90.3 & $2.2(0-7)$ & \multirow[t]{4}{*}{0.0712} & 46 & 16 & \multirow[t]{4}{*}{0.3921} \\
\hline N1 & 31 & $52.7(54)$ & 28 & 90.3 & $2.9(0-15)$ & & 24 & 7 & \\
\hline N2 & 6 & $46.7(43)$ & 6 & 100 & $2(2)$ & & 6 & 0 & \\
\hline N3 & 3 & $58.3(55)$ & 3 & 100 & $5.3(3-9)$ & & 3 & 0 & \\
\hline \multicolumn{10}{|c|}{ Lymph Node Metastasis } \\
\hline Yes & 40 & $52.3(54)$ & 37 & 92.5 & $3(0-15)$ & \multirow[t]{2}{*}{0.1673} & 33 & 7 & \multirow[t]{2}{*}{0.3269} \\
\hline No & 62 & $54.9(56)$ & 56 & 90.3 & $2.2(0-7)$ & & 46 & 16 & \\
\hline \multicolumn{10}{|c|}{ Molecular Subtypes } \\
\hline $\begin{array}{l}\text { Luminal } \\
\mathrm{A}\end{array}$ & 34 & $53.9(55)$ & 29 & 85.3 & $2.3(0-9)$ & \multirow[t]{4}{*}{0.8058} & 25 & 9 & \multirow[t]{4}{*}{0.4713} \\
\hline $\begin{array}{l}\text { Luminal } \\
B\end{array}$ & 36 & $52.5(54.5)$ & 34 & 94.4 & $2.9(0-15)$ & & 31 & 5 & \\
\hline $\begin{array}{l}\text { HER2 } \\
\text { OE }\end{array}$ & 12 & $53.8(54.5)$ & 12 & 100 & $2.4(1-5)$ & & 9 & 3 & \\
\hline TN & 20 & $55.3(54.5)$ & 18 & 90 & $2.1(0-5)$ & & 14 & 6 & \\
\hline \multicolumn{10}{|c|}{ Histology Grade(1 = low; 2 = medium; 3 = high $)(\mathrm{n}=63)$} \\
\hline 1 & 6 & $55.2(56.5)$ & 6 & 100 & $2.2(1-3)$ & \multirow[t]{2}{*}{0.9832} & 5 & 1 & \multirow[t]{2}{*}{0.029} \\
\hline 2 & 40 & $54.6(56.5)$ & 37 & 92.5 & $2.5(0-15)$ & & 34 & 6 & \\
\hline
\end{tabular}




\section{3}

$17 \quad 53.5(53)$

16

94.1

$2.2(0-5)$

12

5

$\mathrm{BC}=$ breast cancer; $\mathrm{n}$ = number of patients; $\mathrm{CTCs}$ = circulating tumor cells; $\mathrm{BBD}$ = benign breast disease; $\mathrm{AJCC}=$ American Joint Committee on Cancer; TNM = tumor-node-metastasis: $\mathrm{OE}$ = over-expression; $\mathrm{TN}$ =triple negative.

Table 2. Correlations of CTCs with ultrasonography in BC 


\begin{tabular}{|c|c|c|c|c|c|c|c|c|c|}
\hline Group & $\mathrm{n}$ & $\begin{array}{l}\text { Average Age } \\
\text { (Median, } \\
\text { years) }\end{array}$ & $\begin{array}{l}\text { CTC } \\
\text { Detected }\end{array}$ & $\begin{array}{l}\text { CTC } \\
\text { Detection } \\
\text { Rate (\%) }\end{array}$ & $\begin{array}{l}\text { Average } \\
\text { CTCs Count } \\
\text { (Range) }\end{array}$ & $P$ & $\begin{array}{l}\text { CTCs } \\
\geq 2\end{array}$ & $\begin{array}{l}\text { CTCs } \\
<2\end{array}$ & $P$ \\
\hline \multicolumn{10}{|c|}{$\boldsymbol{U S}(\mathrm{n}=101)^{*}$} \\
\hline $1 \mathrm{a}$ & 3 & $58.3(57)$ & 3 & 100 & $1.7(1-2)$ & \multirow[t]{5}{*}{0.0639} & 2 & 1 & \multirow[t]{5}{*}{0.5181} \\
\hline $1 b$ & 8 & $55.6(57)$ & 7 & 87.5 & $1.6(0-3)$ & & 5 & 3 & \\
\hline $1 c$ & 38 & $51(51)$ & 36 & 94.7 & $2.3(0-7)$ & & 32 & 6 & \\
\hline 2 & 49 & $55.2(55)$ & 43 & 87.8 & $2.8(0-15)$ & & 37 & 12 & \\
\hline 3 & 3 & $56.3(60)$ & 3 & 100 & 4 & & 3 & 0 & \\
\hline $1 a+1 b$ & 11 & $56.4(57)$ & 10 & 90.9 & $1.6(0-3)$ & \multirow[t]{4}{*}{0.0309} & 7 & 4 & \multirow[t]{4}{*}{0.3589} \\
\hline $1 c$ & 38 & $51(51)$ & 36 & 94.7 & $2.3(0-7)$ & & 32 & 6 & \\
\hline 2 & 49 & $55.2(55)$ & 43 & 87.8 & $2.8(0-15)$ & & 37 & 12 & \\
\hline 3 & 3 & $56.3(60)$ & 3 & 100 & 4 & & 3 & 0 & \\
\hline 1 & 49 & $52.2(53)$ & 46 & 93.9 & $2.1(0-7)$ & \multirow[t]{3}{*}{0.0322} & 39 & 10 & \multirow[t]{3}{*}{0.5768} \\
\hline 2 & 49 & $55.2(55)$ & 43 & 87.8 & $2.8(0-15)$ & & 37 & 12 & \\
\hline 3 & 3 & $56.3(60)$ & 3 & 100.0 & 4 & & 3 & 0 & \\
\hline \multicolumn{10}{|c|}{ US BI-RADS $(\mathrm{n}=101+240)$} \\
\hline 1 & 64 & $40.4(36)$ & 12 & 18.8 & $0.2(0-1)$ & \multirow{8}{*}{$\hat{\delta} .0001$} & 0 & 64 & \multirow{8}{*}{$\begin{array}{l}<.0001 \\
0.00\end{array}$} \\
\hline 2 & 7 & $53(52)$ & 3 & 42.9 & $0.4(0-1)$ & & 0 & 7 & \\
\hline 3 & 82 & $39.2(39)$ & 25 & 30.5 & $0.4(0-4)$ & & 7 & 75 & \\
\hline $4 a$ & 71 & $46.8(46)$ & 41 & 57.7 & $0.8(0-6)$ & & 12 & 59 & \\
\hline $4 b$ & 54 & $49.9(49.5)$ & 36 & 66.7 & $1.1(0-3)$ & & 21 & 33 & \\
\hline $4 c$ & 31 & $53.7(55)$ & 27 & 87.1 & $2.9(0-15)$ & & 25 & 6 & \\
\hline 5 & 31 & $56.7(58)$ & 29 & 93.5 & $2.8(0-9)$ & & 28 & 3 & \\
\hline 6 & 1 & 32 & 1 & 100.0 & 2 & & 1 & 0 & \\
\hline 1 & 64 & $40.4(36)$ & 12 & 18.8 & $0.2(0-1)$ & \multirow{6}{*}{$\begin{array}{l}<.0001 \\
\end{array}$} & 0 & 64 & \multirow{6}{*}{ <. 0001} \\
\hline 2 & 7 & $53(52)$ & 3 & 42.9 & $0.4(0-1)$ & & 0 & 7 & \\
\hline 3 & 82 & $39.2(39)$ & 25 & 30.5 & $0.4(0-4)$ & & 7 & 75 & \\
\hline 4 & 156 & $49.2(49)$ & 104 & 66.7 & $1.4(0-15)$ & & 58 & 98 & \\
\hline 5 & 31 & $56.9(57)$ & 29 & 93.5 & $2.9(0-9)$ & & 28 & 3 & \\
\hline 6 & 1 & 32 & 1 & 100.0 & 2 & & 1 & 0 & \\
\hline$\leqq 4 a$ & 224 & $42.4(42)$ & 81 & 36.2 & $0.5(0-6)$ & \multirow{2}{*}{$\begin{array}{l}<.0001 \\
\end{array}$} & 19 & 205 & \multirow{2}{*}{$\overleftarrow{0} .0001$} \\
\hline$\geqq 4 b$ & 117 & $52.5(54)$ & 93 & 79.5 & $2.1(0-15)$ & & 75 & 42 & \\
\hline
\end{tabular}


CTCs = circulating tumor cells; $\mathrm{BC}$ = breast cancer; $\mathrm{n}$ = number of patients; $\mathrm{US}=$ ultrasonography; $\mathrm{BI}$-RADS = breast imaging reporting and data system.

* Based on the tumor size $T$ estimated by imaging, five categories were defined - 1a, 1b, 1c, 2 and 3, representing patients with $T \leq 0.5 \mathrm{~cm}, 0.5 \mathrm{~cm}<T \leq 1 \mathrm{~cm}, 1 \mathrm{~cm}<T \leq 2 \mathrm{~cm}, 2 \mathrm{~cm}<T \leq 5 \mathrm{~cm}$, and $T>5 \mathrm{~cm}$, respectively.

Table 3. Correlations of CTCs with mammography in BC 


\begin{tabular}{|c|c|c|c|c|c|c|c|c|c|}
\hline Group & $\mathrm{n}$ & $\begin{array}{l}\text { Average Age } \\
\text { (Median, } \\
\text { years) }\end{array}$ & $\begin{array}{l}\text { CTC } \\
\text { Detected }\end{array}$ & $\begin{array}{l}\text { CTC } \\
\text { Detection } \\
\text { Rate (\%) }\end{array}$ & $\begin{array}{l}\text { Average } \\
\text { CTCs Count } \\
\text { (Range) }\end{array}$ & $P$ & $\begin{array}{l}\text { CTCs } \\
\geq 2\end{array}$ & $\begin{array}{l}\text { CTCs } \\
<2\end{array}$ & $P$ \\
\hline \multicolumn{10}{|c|}{$\boldsymbol{M} \boldsymbol{G}(\mathrm{n}=43)^{\star}$} \\
\hline $1 a$ & 1 & 62 & 1 & 100 & 3 & \multirow[t]{5}{*}{0.568} & 1 & 0 & \multirow[t]{5}{*}{0.5951} \\
\hline $1 b$ & 5 & $57.2(59)$ & 3 & 60 & $1.4(0-3)$ & & 3 & 2 & \\
\hline $1 \mathrm{c}$ & 13 & $54.9(56)$ & 12 & 92.3 & $2.4(0-6)$ & & 11 & 2 & \\
\hline 2 & 22 & $56.6(55)$ & 20 & 90.9 & $2.2(0-9)$ & & 14 & 8 & \\
\hline 3 & 2 & $60(60)$ & 1 & 50 & $2(0-4)$ & & 1 & 1 & \\
\hline $1 a+1 b$ & 6 & $58(60)$ & 10 & 166.7 & $1.7(0-3)$ & \multirow[t]{4}{*}{0.7507} & 4 & 2 & \multirow[t]{4}{*}{0.5421} \\
\hline $1 \mathrm{c}$ & 13 & $54.9(56)$ & 36 & 276.9 & $2.4(0-6)$ & & 11 & 2 & \\
\hline 2 & 22 & $56.6(55)$ & 43 & 195.5 & $2.2(0-9)$ & & 14 & 8 & \\
\hline 3 & 2 & $60(60)$ & 3 & 100 & $2(0-4)$ & & 1 & 1 & \\
\hline 1 & 19 & $55.9(56)$ & 46 & 242.1 & $2.2(0-6)$ & \multirow[t]{3}{*}{0.7807} & 15 & 4 & \multirow[t]{3}{*}{0.4672} \\
\hline 2 & 22 & $56.6(55)$ & 43 & 195.5 & $2.2(0-9)$ & & 14 & 8 & \\
\hline 3 & 2 & $60(60)$ & 3 & 150.0 & $2(0-4)$ & & 1 & 1 & \\
\hline \multicolumn{10}{|c|}{ MG Breast Tissue Composition Categories $(\mathrm{n}=82)$} \\
\hline a & 8 & $62.9(61)$ & 8 & 100 & $2.4(1-4)$ & \multirow[t]{4}{*}{0.6793} & 6 & 2 & \multirow[t]{4}{*}{0.4119} \\
\hline b & 12 & $60.8(59)$ & 11 & 91.7 & $2.6(0-9)$ & & 9 & 3 & \\
\hline c & 52 & $51.3(50)$ & 47 & 90.4 & $2.6(0-15)$ & & 42 & 10 & \\
\hline$d$ & 10 & $49(48)$ & 10 & 100 & $3.1(2-7)$ & & 10 & 0 & \\
\hline$a+b$ & 20 & $61.7(60.5)$ & 19 & 95 & $2.5(0-9)$ & \multirow[t]{2}{*}{0.8808} & 17 & 5 & \multirow[t]{2}{*}{0.5244} \\
\hline$c+d$ & 62 & $50.9(50)$ & 57 & 91.9 & $2.7(0-15)$ & & 52 & 10 & \\
\hline \multicolumn{10}{|c|}{ MG BI-RADS $(\mathrm{n}=72+106)$} \\
\hline 1 & 6 & $45.6(45)$ & 2 & 33.3 & $0.4(0-1)$ & \multirow{7}{*}{$\hat{0} .0001$} & 0 & 6 & \multirow{7}{*}{$\dot{0} 0001$} \\
\hline 2 & 34 & $47.2(45.5)$ & 11 & 32.4 & $0.4(0-3)$ & & 3 & 31 & \\
\hline 3 & 52 & $50.6(51)$ & 29 & 55.8 & $0.9(0-3)$ & & 13 & 39 & \\
\hline $4 a$ & 26 & $50(49)$ & 15 & 57.7 & $0.9(0-4)$ & & 5 & 21 & \\
\hline $4 \mathrm{~b}$ & 24 & $49(49)$ & 18 & 75.0 & $2.3(0-15)$ & & 15 & 9 & \\
\hline $4 c$ & 31 & $55.9(59)$ & 28 & 90.3 & $2.5(0-9)$ & & 24 & 7 & \\
\hline 5 & 5 & $59(58)$ & 5 & 100.0 & $2.2(1-3)$ & & 4 & 1 & \\
\hline 1 & 6 & $45.6(45)$ & 2 & 33.3 & $0.4(0-1)$ & \multirow{2}{*}{$\overleftarrow{0}_{0.0001}$} & 0 & 6 & \multirow{2}{*}{$\hat{0} 00001$} \\
\hline 2 & 34 & $47.2(45.5)$ & 11 & 32.4 & $0.4(0-3)$ & & 3 & 31 & \\
\hline
\end{tabular}




\begin{tabular}{|c|c|c|c|c|c|c|c|c|c|}
\hline 3 & 52 & $50.6(51)$ & 29 & 55.8 & $0.9(0-3)$ & & 13 & 39 & \\
\hline 4 & 81 & $51.9(53)$ & 61 & 75.3 & $1.9(0-15)$ & & 44 & 37 & \\
\hline 5 & 5 & $59(58)$ & 5 & 100 & $2.2(1-3)$ & & 4 & 1 & \\
\hline$\leqq 4 a$ & 118 & $49.1(49)$ & 57 & 86.4 & $0.7(0-4)$ & \multirow{2}{*}{$\hat{0} .0001$} & 21 & 97 & \multirow{2}{*}{$\hat{0} .0001$} \\
\hline$\geqq 4 b$ & 60 & $56.3(58.5)$ & 51 & 94 & $2.4(0-15)$ & & 43 & 17 & \\
\hline
\end{tabular}

CTCs = circulating tumor cells; $B C=$ breast cancer; $n=$ number of patients; $M G=$ mammography; BI-RADS = breast imaging reporting and data system.

* Based on the tumor size $T$ estimated by imaging, five categories were defined - 1a, 1b, 1c, 2 and 3, representing patients with $T \leq 0.5 \mathrm{~cm}, 0.5 \mathrm{~cm}<T \leq 1 \mathrm{~cm}, 1 \mathrm{~cm}<T \leq 2 \mathrm{~cm}, 2 \mathrm{~cm}<T \leq 5 \mathrm{~cm}$, and $T>5 \mathrm{~cm}$, respectively.

Table 4. Correlations of CTCs with magnetic resonance imaging examination in BC 


\begin{tabular}{|c|c|c|c|c|c|c|c|c|c|}
\hline Group & $\mathrm{n}$ & $\begin{array}{l}\text { Average } \\
\text { Age } \\
\text { (Median, } \\
\text { years) }\end{array}$ & $\begin{array}{l}\text { CTC } \\
\text { Detected }\end{array}$ & $\begin{array}{l}\text { CTC } \\
\text { Detection } \\
\text { Rate (\%) }\end{array}$ & $\begin{array}{l}\text { Average } \\
\text { CTCs } \\
\text { Count } \\
\text { (Range) }\end{array}$ & $P$ & $\begin{array}{l}\text { CTCs } \\
\geq 2\end{array}$ & $\begin{array}{l}\text { CTCs } \\
<2\end{array}$ & $P$ \\
\hline \multicolumn{10}{|c|}{$M R(\mathrm{n}=83)^{*}$} \\
\hline $1 a$ & 2 & $\begin{array}{l}58.5 \\
(58.5)\end{array}$ & 2 & 100 & $1.5(1-2)$ & \multirow[t]{5}{*}{0.1843} & 1 & 1 & \multirow[t]{5}{*}{0.2827} \\
\hline $1 b$ & 4 & $50.5(52)$ & 3 & 75 & $2.8(0-6)$ & & 3 & 1 & \\
\hline $1 \mathrm{c}$ & 36 & $53.7(54)$ & 31 & 86.1 & $1.8(0-4)$ & & 26 & 10 & \\
\hline 2 & 35 & $51.7(49)$ & 33 & 94.3 & $2.6(0-9)$ & & 30 & 5 & \\
\hline 3 & 6 & $\begin{array}{l}57.2 \\
(59.5)\end{array}$ & 6 & 100 & $2.2(1-4)$ & & 3 & 3 & \\
\hline $1 a+1 b$ & 6 & $\begin{array}{l}53.2 \\
(56.5)\end{array}$ & 5 & 83.3 & $2.3(0-6)$ & \multirow[t]{4}{*}{0.167} & 4 & 2 & \multirow[t]{4}{*}{0.2044} \\
\hline $1 \mathrm{c}$ & 36 & $53.7(54)$ & 31 & 86.1 & $1.8(0-4)$ & & 26 & 10 & \\
\hline 2 & 35 & $51.7(49)$ & 33 & 94.3 & $2.6(0-9)$ & & 30 & 5 & \\
\hline 3 & 6 & $\begin{array}{l}57.2 \\
(59.5)\end{array}$ & 6 & 100 & $2.2(1-4)$ & & 3 & 3 & \\
\hline 1 & 42 & $\begin{array}{l}53.6 \\
(54.5)\end{array}$ & 36 & 85.7 & $1.9(0-6)$ & \multirow[t]{3}{*}{0.0876} & 30 & 12 & \multirow[t]{3}{*}{0.1052} \\
\hline 2 & 35 & $51.7(49)$ & 33 & 94.3 & $2.6(0-9)$ & & 30 & 5 & \\
\hline 3 & 6 & $\begin{array}{l}57.2 \\
(59.5)\end{array}$ & 6 & 100 & $2.2(1-4)$ & & 3 & 3 & \\
\hline \multicolumn{10}{|c|}{ MR Breast Tissue Composition Categories $(\mathrm{n}=91)$} \\
\hline a & 3 & $58(66)$ & 1 & 33.3 & $2.4(1-4)$ & \multirow[t]{4}{*}{0.683} & 1 & 2 & \multirow[t]{4}{*}{0.1837} \\
\hline $\mathrm{b}$ & 27 & $60.3(60)$ & 24 & 88.9 & $2.6(0-9)$ & & 19 & 8 & \\
\hline c & 52 & $51.2(50)$ & 49 & 94.2 & $2.6(0-15)$ & & 43 & 9 & \\
\hline$d$ & 9 & $45.1(43)$ & 9 & 100 & $3.1(2-7)$ & & 7 & 2 & \\
\hline$a+b$ & 30 & $60.1(60)$ & 25 & 83.3 & $2.5(0-9)$ & \multirow[t]{2}{*}{0.4016} & 20 & 10 & \multirow[t]{2}{*}{0.1034} \\
\hline$c+d$ & 61 & $50.3(48)$ & 58 & 95.1 & $2.7(0-15)$ & & 50 & 11 & \\
\hline \multicolumn{10}{|c|}{ MR BI-RADS $(\mathrm{n}=86+6)$} \\
\hline 3 & 7 & $51.9(49)$ & 6 & 85.7 & $2.1(0-6)$ & \multirow[t]{6}{*}{0.0267} & 4 & 3 & \multirow[t]{6}{*}{0.0123} \\
\hline $4 a$ & 9 & $41.7(50)$ & 6 & 67 & $0.9(0-2)$ & & 2 & 7 & \\
\hline $4 b$ & 15 & $51.9(49)$ & 15 & 100 & $2.2(1-7)$ & & 11 & 4 & \\
\hline $4 c$ & 31 & $52.3(55)$ & 27 & 87 & $2.7(0-15)$ & & 24 & 7 & \\
\hline 5 & 15 & $52.8(54)$ & 15 & 100 & $2.5(1-5)$ & & 14 & 1 & \\
\hline 6 & 15 & $54.1(54)$ & 13 & 86.7 & $\begin{array}{l}2.4(0-5) \\
19 / 21\end{array}$ & & 11 & 4 & \\
\hline
\end{tabular}




\begin{tabular}{|c|c|c|c|c|c|c|c|c|c|}
\hline 3 & 7 & $51.9(49)$ & 6 & 85.7 & $2.1(0-6)$ & \multirow[t]{4}{*}{0.3961} & 4 & 3 & \multirow[t]{4}{*}{0.1962} \\
\hline 4 & 55 & $51.1(52)$ & 48 & 87.3 & $2.3(0-15)$ & & 37 & 18 & \\
\hline 5 & 15 & $52.8(54)$ & 15 & 100 & $2.5(1-5)$ & & 14 & 1 & \\
\hline 6 & 15 & $54.1(54)$ & 13 & 86.7 & $2.4(0-5)$ & & 11 & 4 & \\
\hline$\leqq 4 a$ & 16 & $\begin{array}{l}48.3 \\
(49.5)\end{array}$ & 9 & 81.8 & $1.4(0-6)$ & \multirow[t]{2}{*}{0.0038} & 6 & 10 & \multirow[t]{2}{*}{0.0018} \\
\hline$\geqq 4 b$ & 76 & $52.7(54)$ & 70 & 93.3 & $2.5(0-15)$ & & 60 & 16 & \\
\hline \multicolumn{10}{|c|}{$M R T I C(\mathrm{n}=91)$} \\
\hline 1 & 22 & $52.4(54)$ & 20 & 90.9 & $2.7(0-15)$ & 0.84 & 16 & 6 & \multirow[t]{3}{*}{0.7251} \\
\hline 2 & 38 & $53.1(53)$ & 36 & 94.7 & $2.3(0-7)$ & & 31 & 7 & \\
\hline 3 & 27 & $55.5(56)$ & 23 & 85.2 & $2.5(0-9)$ & & 21 & 6 & \\
\hline
\end{tabular}

CTCs = circulating tumor cells; $\mathrm{BC}=$ breast cancer; $\mathrm{n}=$ number of patients; $\mathrm{MR}=$ magnetic resonance imaging; $\mathrm{BI}-$ RADS = breast imaging reporting and data system; TIC = time-signal intensity curves.

* Based on the tumor size $T$ estimated by imaging, five categories were defined - 1a, 1b, 1c, 2 and 3, representing patients with $T \leq 0.5 \mathrm{~cm}, 0.5 \mathrm{~cm}<T \leq 1 \mathrm{~cm}, 1 \mathrm{~cm}<T \leq 2 \mathrm{~cm}, 2 \mathrm{~cm}<T \leq 5 \mathrm{~cm}$, and $T>5 \mathrm{~cm}$, respectively.

Table 5. Correlations of CTCs with breast-specific gamma-imaging in BC

\begin{tabular}{|llllll|}
\hline Group & $\mathrm{n}$ & $\begin{array}{l}\text { Average Age (Median, } \\
\text { years) }\end{array}$ & $\begin{array}{l}\text { CTC } \\
\text { Detected }\end{array}$ & $\begin{array}{l}\text { CTC Detection Rate } \\
(\%)\end{array}$ & $\begin{array}{l}\text { Average CTC Count } \\
\text { (Range) }\end{array}$ \\
\hline BSGI Score $(\mathbf{1 . 8 2} n)(\mathrm{n}=42)$ & & 6 & 100 & $2.3(2-3)$ \\
\hline 1 & 6 & $54.5(53.5)$ & 60 & 100 & $2.6(1-13)$ \\
\hline 2 & 30 & $53.5(54)$ & 30 & 100 & $2(2-15)$ \\
\hline 3 & 6 & $52(52)$ & 6 & 64 \\
\hline
\end{tabular}

CTCs = circulating tumor cells; $\mathrm{BC}=$ breast cancer; $\mathrm{n}$ = number of patients; $\mathrm{BSGI}$ = breast-specific gamma-imaging

Table 6. Correlations of CTCs and medical imaging examinations with BC patients' clinicopathological features with cut-off setting* 


\begin{tabular}{|lllll|}
\hline Characteristics & $P$ & & & \\
\cline { 2 - 5 } & CTC $(\mathrm{n}=102)$ & US $(\mathrm{n}=101)$ & $\mathrm{MG}(\mathrm{n}=72)$ & MR $(\mathrm{n}=76)$ \\
\hline BC versus Control & $<\mathbf{0 . 0 0 0 1}$ & $<\mathbf{0 . 0 0 0 1}$ & $<\mathbf{0 . 0 0 0 1}$ & $\mathbf{0 . 0 0 0 8}$ \\
\hline AJCC Stage & 0.0964 & $\mathbf{0 . 0 4 3 8}$ & 0.3332 & $\mathbf{0 . 0 4 2 2}$ \\
\hline TNM T Stage & 0.2417 & 0.1923 & 0.1668 & 0.116 \\
\hline TNM N Stage & 0.3921 & 0.0865 & 0.6971 & 0.422 \\
\hline Lymph Node Metastasis & 0.3269 & $\mathbf{0 . 0 1 5 7}$ & 0.2609 & 0.0747 \\
\hline Molecular Subtypes & 0.4713 & 0.9188 & 0.8881 & 0.088 \\
\hline
\end{tabular}

CTCs = circulating tumor cells; $\mathrm{BC}$ = breast cancer; $\mathrm{n}$ = number of patients; $\mathrm{US}=$ ultrasonography; $\mathrm{MG}=$ mammography; MR = magnetic resonance imaging.

* CTCs is set to 2 , while medical imaging BI-RADS is set to $4 \mathrm{~b}$.

Table 7. Diagnostic performances of CTCs and medical imaging examinationa in BC diagnosis.

\begin{tabular}{|c|c|c|c|c|c|c|c|c|c|c|}
\hline Method & $\begin{array}{l}\text { n (BC+ } \\
\text { Control) }\end{array}$ & $\begin{array}{l}\text { Cut- } \\
\text { off }\end{array}$ & Sensitivity & Specificity & PPV & NPV & FPR & FNR & Accuracy & AUC \\
\hline СТC & $\begin{array}{l}102+ \\
241\end{array}$ & 2 & 0.775 & 0.938 & 0.840 & 0.908 & 0.062 & 0.225 & 0.889 & 0.894 \\
\hline US & $\begin{array}{l}101+ \\
240\end{array}$ & $4 b$ & 0.842 & 0.850 & 0.702 & 0.927 & 0.150 & 0.158 & 0.848 & 0.927 \\
\hline MG & $\begin{array}{l}72+ \\
106\end{array}$ & $4 b$ & 0.694 & 0.906 & 0.833 & 0.814 & 0.094 & 0.306 & 0.820 & 0.855 \\
\hline MR & $86+6$ & $4 b$ & 0.872 & 0.833 & 0.987 & 0.313 & 0.167 & 0.128 & 0.870 & 0.841 \\
\hline $\begin{array}{l}\text { CTC + } \\
\text { US }\end{array}$ & $\begin{array}{l}101+ \\
240\end{array}$ & $\begin{array}{l}2 / \\
4 \mathrm{~b}\end{array}$ & 0.921 & 0.821 & 0.684 & 0.961 & 0.179 & 0.079 & 0.850 & 0.958 \\
\hline $\begin{array}{l}\text { CTC + } \\
\text { MG }\end{array}$ & $\begin{array}{l}72+ \\
106\end{array}$ & $\begin{array}{l}2 / \\
4 \mathrm{~b}\end{array}$ & 0.917 & 0.858 & 0.815 & 0.938 & 0.142 & 0.083 & 0.882 & 0.950 \\
\hline $\begin{array}{l}\text { CTC + } \\
\text { MR }\end{array}$ & $86+6$ & $\begin{array}{l}2 / \\
4 \mathrm{~b}\end{array}$ & 0.942 & 0.833 & 0.988 & 0.500 & 0.167 & 0.058 & 0.935 & 0.955 \\
\hline $\begin{array}{l}\text { US + } \\
\text { MG }\end{array}$ & $\begin{array}{l}71+ \\
106\end{array}$ & $4 b$ & 0.901 & 0.736 & 0.696 & 0.918 & 0.264 & 0.099 & 0.802 & 0.915 \\
\hline $\begin{array}{l}\text { US + } \\
\text { MR }\end{array}$ & $85+5$ & $4 b$ & 0.941 & 0.400 & 0.964 & 0.286 & 0.600 & 0.059 & 0.911 & 0.903 \\
\hline $\begin{array}{l}\text { MG + } \\
\text { MR }\end{array}$ & $60+2$ & $4 b$ & 0.950 & 0.500 & 0.983 & 0.250 & 0.500 & 0.050 & 0.935 & 0.918 \\
\hline
\end{tabular}

CTCs = circulating tumor cells; $\mathrm{n}$ = number of patients; $\mathrm{BC}=$ breast cancer; $\mathrm{PPV}=$ positive predictive value; NPV = negative predictive value; $F P R=$ false positive rate; $F N R=$ false negative rate; $A U C$ = area under curve; US = ultrasonography; $\mathrm{MG}=$ mammography; $\mathrm{MR}=$ magnetic resonance imaging. 